\title{
New Rule for Choice of the Regularization Parameter in (Iterated) Tikhonov Method*
}

\section{T. Raus and U. Hämarik}

Institute of Mathematics, University of Tartu

J. Liivi 2, 50409 Tartu

E-mail(corresp.): toomas.raus@ut.ee

E-mail: uno.hamarik@ut.ee

Received October 16, 2008; revised February 12, 2009; published online April 30, 2009

\begin{abstract}
We propose a new a posteriori rule for choosing the regularization parameter $\alpha$ in (iterated) Tikhonov method for solving linear ill-posed problems in Hilbert spaces. We assume that data are noisy but noise level $\delta$ is given. We prove that (iterated) Tikhonov approximation with proposed choice of $\alpha$ converges to the solution as $\delta \rightarrow 0$ and has order optimal error estimates. Under certain mild assumption the quasioptimality of proposed rule is also proved. Numerical examples show the advantage of the new rule over the monotone error rule, especially in case of rough $\delta$.
\end{abstract}

Key words: ill-posed problem, (iterated) Tikhonov method, regularization parameter, a posteriori rule, monotone error rule, convergence, order optimality.

\section{Introduction}

In this paper we consider linear ill-posed problems

$$
A u=f,
$$

where $A \in L(H, F)$ is a bounded operator with non-closed range $R(A)$ of $A$ and $H, F$ are infinite-dimensional real Hilbert spaces. We assume that instead of exact data $f$ there are given noisy data $f_{\delta} \in F$ with $\left\|f_{\delta}-f\right\| \leq \delta$ and known noise level $\delta$. The well-known methods for solving ill-posed problems are the Tikhonov method

$$
u_{\alpha}=\left(\alpha I+A^{*} A\right)^{-1} A^{*} f_{\delta}
$$

and iterated variant of this method

$$
u_{\alpha}=u_{\alpha, m}, \quad u_{\alpha, i}=\left(\alpha I+A^{*} A\right)^{-1}\left(\alpha u_{\alpha, i-1}+A^{*} f_{\delta}\right), \quad(i=1,2, \ldots, m) .
$$

\footnotetext{
* This work was supported by the Estonian Science Foundation, Research Grant No. 7489
} 
Note that approximation (1.2) is a special case of (1.3), where $m=1$ and for initial approximation $u_{\alpha, 0}=0$ is used. For applying methods (1.2), (1.3) the important problem is a proper choice of the regularization parameter $\alpha=\alpha(\delta)$.

Well-known rules for parameter choice in methods (1.2), (1.3) are the discrepancy principle $[8,19,20]$, the modified discrepancy (MD) rule $[1,11,12]$, the monotone error $(\mathrm{ME})$ rule $[18]$, rule $\mathrm{R} 1([13,14,15])$. In this paper we propose a new rule. In numerical experiments we get typically smaller error of approximations $(1.2),(1.3)$ than by using the other rules, particularly in case of rough estimates of the noise level. Both, the rule $\mathrm{R} 1$ and the proposed rule are applicable also in the case, when problem (1.1) has solution only in the least squares sense $\left(f \notin \mathcal{R}(A)\right.$, but $\left.A^{*} f \in \mathcal{R}\left(A^{*} A\right)\right)$.

In this paper in Section 2 we consider rules for a posteriori choice of $\alpha$. For the proposed rule the convergence and order optimality are proved in Section 3. The quasioptimality properties of the new rule are studied in Section 4. In the final section numerical results are given.

\section{Rules for Parameter Choice}

Before considering parameter choice rules we remind, that approximations (1.2), (1.3) have the following form

$$
u_{\alpha}=u_{0}+g_{\alpha}\left(A^{*} A\right) A^{*}\left(f_{\delta}-A u_{0}\right),
$$

where the generating function

$$
g_{\alpha, m}(\lambda)=\frac{1}{\lambda}\left[1-\left(\frac{\alpha}{\alpha+\lambda}\right)^{m}\right]
$$

satisfies conditions

$$
\begin{aligned}
& \sup _{0 \leq \lambda \leq\left\|A^{*} A\right\|}\left|\sqrt{\lambda} g_{\alpha}(\lambda)\right| \leq \gamma_{*} \alpha^{-1 / 2}, \quad \alpha>0, \\
& \sup _{0 \leq \lambda \leq\left\|A^{*} A\right\|} \lambda^{p}\left|1-\lambda g_{\alpha}(\lambda)\right| \leq \gamma_{p} \alpha^{p}, \quad 0 \leq p \leq m .
\end{aligned}
$$

Here $\gamma_{p}=\left(\frac{p}{m}\right)^{p}\left(1-\frac{p}{m}\right)^{m-p}$ and $\gamma_{*}=1 / 2$ for the Tikhonov method and $\gamma_{*}=\sqrt{m}$ in case $m \geq 2$.

We introduce notations

$$
\begin{aligned}
& B_{\alpha}=\alpha^{1 / 2}\left(\alpha I+A A^{*}\right)^{-1 / 2}, \quad \bar{B}_{\alpha}=\alpha^{1 / 2}\left(\alpha I+A^{*} A\right)^{-1 / 2}, \\
& D_{\alpha}=\alpha^{-1}\left|A^{*}\right|^{2} B_{\alpha}^{2}, \quad\left|A^{*}\right|=\left(A A^{*}\right)^{1 / 2} .
\end{aligned}
$$

In the following we consider rules for the choice of the regularization parameter $\alpha=\alpha(\delta)$ for guaranteeing the convergence of the regularized solution

$$
\left\|u_{\alpha(\delta)}-u_{*}\right\| \rightarrow 0 \quad \text { as } \quad \delta \rightarrow 0,
$$

where $u_{*}$ is a solution of problem (1.1) nearest to the initial approximation $u_{0}$. 
Let $b=$ const $\geq 1$. Then in the discrepancy principle, in MD rule and in ME rule the corresponding regularization parameters $\alpha_{D}, \alpha_{M D}$ and $\alpha_{M E}$ are chosen as solutions $\alpha=\alpha(\delta)$ of equations

$$
\begin{aligned}
& \left\|A u_{\alpha}-f_{\delta}\right\|=b \delta \\
& \| B_{\alpha}\left(A u_{\alpha}-f_{\delta} \|:=\left(A u_{\alpha, m+1}-f_{\delta}, A u_{\alpha, m}-f_{\delta}\right)^{1 / 2}=b \delta\right. \\
& \frac{\| B_{\alpha}\left(A u_{\alpha}-f_{\delta} \|^{2}\right.}{\| B_{\alpha}^{2}\left(A u_{\alpha}-f_{\delta} \|\right.}=\frac{\left(A u_{\alpha, m}-f_{\delta}, A u_{\alpha, m+1}-f_{\delta}\right)}{\left\|A u_{\alpha, m+1}-f_{\delta}\right\|}=b \delta
\end{aligned}
$$

respectively. The ME rule has the properties

$$
\left\|u_{\alpha_{M E}}-u_{*}\right\| \leq\left\|u_{\alpha_{M D}}-u_{*}\right\|, \quad \frac{d}{d \alpha}\left\|u_{\alpha}-u_{*}\right\| \geq 0 \quad \forall \alpha \in\left(\alpha_{M E}, \infty\right),
$$

therefore $\alpha_{M E} \geq \alpha_{o p t}:=\arg \min \left\{\left\|u_{\alpha}-u_{*}\right\|, \alpha>0\right\}$. Usually $\alpha_{M E}<1$ and $\alpha_{o p t}=\alpha_{M E}^{c}$ with some $c \geq 1$.

Our extensive numerical experiments presented in [2] have suggested the use of MEE rule with parameter $\alpha_{M E E}=\alpha_{M E}^{1.1}$ and we have obtained the average error ratio $\left\|u_{\alpha_{M E E}}-u_{*}\right\| /\left\|u_{\alpha_{M E}}-u_{*}\right\| \approx 0.8$.

We remind also rule $\mathrm{R} 1$ with $k=1 / 2$ (see [15]).

Rule R1. Let $b_{2}, b_{1}$ be the constants with $b_{2} \geq b_{1}>\gamma_{m /(2(m+1))}^{(m+1) / m}$. Let $\varphi(\alpha):=\left\|D_{\alpha}^{1 / 2} B_{\alpha}\left(A u_{\alpha}-f_{\delta}\right)\right\|$. Choose $\alpha(\delta)$ such that $\varphi(\alpha(\delta)) \geq b_{1} \delta$ and $\varphi(\alpha) \leq b_{2} \delta$ for all $\alpha \leq \alpha(\delta)$.

Note that in [3] a modification of rule R1 is given, which guarantees convergence $\left\|u_{\alpha(\delta)}-u_{*}\right\| \rightarrow 0(\delta \rightarrow 0)$ also in case $\left\|f_{\delta}-f\right\| / \delta \leq c$ for $\delta \rightarrow 0$, where $c$ is an unknown constant.

If $u_{*}-u_{0} \in \mathcal{R}\left(\left(A^{*} A\right)^{p / 2}\right)$, the order optimal error estimate $\left\|u_{*}-u_{\alpha}\right\|=$ $\mathcal{O}\left(\delta^{p(p+1)}\right)$ holds true for the range $p \in(0,2 m-1]$, if $\alpha$ is chosen by the discrepancy principle, and for the larger range $p \in(0,2 m]$, if $\alpha$ is chosen by the MD, ME or by the $\mathrm{R} 1$ rule.

To introduce a new rule, we denote

$$
\begin{aligned}
\kappa_{\alpha}:= & \left(1+\alpha\|A\|^{-2}\right)^{1 / 2}, \quad \widetilde{\gamma}_{k, s}:=\frac{s^{s / 2}(2 k)^{k}}{(s+2 k)^{k+s / 2}}, \\
d(\alpha):= & \frac{\kappa_{\alpha}\left\|D_{\alpha}^{1 / 2} B_{\alpha}\left(A u_{\alpha}-f_{\delta}\right)\right\|^{2}}{\left\|D_{\alpha}^{1 / 2} B_{\alpha}^{2}\left(A u_{\alpha}-f_{\delta}\right)\right\|}=\frac{\kappa_{\alpha}\left\|A^{*} B_{\alpha}^{2}\left(A u_{\alpha}-f_{\delta}\right)\right\|^{2}}{\sqrt{\alpha}\left\|A^{*} B_{\alpha}^{3}\left(A u_{\alpha}-f_{\delta}\right)\right\|} \\
= & \frac{\kappa_{\alpha}\left\|A^{*}\left(A u_{\alpha, m+1}-f_{\delta}\right)\right\|^{2}}{\sqrt{\alpha}\left\langle A A^{*}\left(A u_{\alpha, m+1}-f_{\delta}\right), A u_{\alpha, m+2}-f_{\delta}\right\rangle^{1 / 2}} .
\end{aligned}
$$

Rule R2. Let $b>\widetilde{\gamma}_{1 / 4, m}^{2}$. We choose the regularization parameter $\alpha=$ $\alpha(\delta)$ as the smallest solution of the equation

$$
d(\alpha)=b \delta
$$


To show that this equation has a solution, let us consider function $d(\alpha)$. It is obvious that

$$
\lim _{\alpha \rightarrow \infty} d(\alpha)=\|A\|^{-1}\left\|A^{*}\left(A u_{0}-f_{\delta}\right)\right\| .
$$

On the other hand, since $\kappa_{\alpha}\left\|D_{\alpha}^{1 / 2}\right\| \leq 1$ and $D_{\alpha}^{1 / 2}=D_{\alpha}^{1 / 2} Q$, where $Q$ is the orthoprojector $F \rightarrow \overline{\mathcal{R}(A)}$, we have

$$
\begin{aligned}
d(\alpha) & =\frac{\kappa_{\alpha}\left\|D_{\alpha}^{1 / 2} B_{\alpha}\left(A u_{\alpha}-f_{\delta}\right)\right\|^{2}}{\left\|D_{\alpha}^{1 / 2} B_{\alpha}^{2}\left(A u_{\alpha}-f_{\delta}\right)\right\|} \leq \frac{\kappa_{\alpha}\left\|D_{\alpha}^{1 / 2}\left(A u_{\alpha}-f_{\delta}\right)\right\|\left\|D_{\alpha}^{1 / 2} B_{\alpha}^{2}\left(A u_{\alpha}-f_{\delta}\right)\right\|}{\left\|D_{\alpha}^{1 / 2} B_{\alpha}^{2}\left(A u_{\alpha}-f_{\delta}\right)\right\|} \\
& =\kappa_{\alpha}\left\|D_{\alpha}^{1 / 2}\left(A u_{\alpha}-f_{\delta}\right)\right\|=\kappa_{\alpha}\left\|D_{\alpha}^{1 / 2} Q\left(A u_{\alpha}-f_{\delta}\right)\right\| \leq\left\|Q\left(A u_{\alpha}-f_{\delta}\right)\right\|,
\end{aligned}
$$

therefore

$$
\left\|Q\left(A u_{\alpha(\delta)}-f_{\delta}\right)\right\| \geq b \delta .
$$

As proved in [14] $\lim _{\alpha \rightarrow 0}\left\|D_{\alpha}^{1 / 2}\left(A u_{\alpha}-f_{\delta}\right)\right\|=0$. On the base of the inequality $d(\alpha) \leq \kappa_{\alpha}\left\|D_{\alpha}^{1 / 2}\left(A u_{\alpha}-f_{\delta}\right)\right\|$ we obtain $\lim _{\alpha \rightarrow 0} d(\alpha)=0$. Therefore in case $\delta \leq\|A\|^{-1}\left\|A^{*}\left(A u_{0}-f_{\delta}\right)\right\|$ the new rule guarantees the choice of $\alpha$.

In order to avoid the instability in computing problems in finding the smallest solution of (2.4) for all $\alpha \geq 0$, we recommend to find the smallest solution for $\alpha \geq\left(\gamma_{*} \delta / M\right)^{2}$, where $M$ is the upper estimate of $\left\|u_{*}-u_{0}\right\|$.

From the following inequality

$$
\begin{aligned}
& d(\alpha)=\frac{\kappa_{\alpha}\left\|D_{\alpha}^{1 / 2} B_{\alpha}\left(A u_{\alpha}-f_{\delta}\right)\right\|^{2}}{\left\|D_{\alpha}^{1 / 2} B_{\alpha}^{2}\left(A u_{\alpha}-f_{\delta}\right)\right\|} \\
& \quad=\kappa_{\alpha}\left\|D_{\alpha}^{1 / 2} B_{\alpha}\left(A u_{\alpha}-f_{\delta}\right)\right\| \frac{\left\|D_{\alpha}^{1 / 2} B_{\alpha}\left(A u_{\alpha}-f_{\delta}\right)\right\|}{\left\|D_{\alpha}^{1 / 2} B_{\alpha}^{2}\left(A u_{\alpha}-f_{\delta}\right)\right\|} \geq \kappa_{\alpha}\left\|D_{\alpha}^{1 / 2} B_{\alpha}\left(A u_{\alpha}-f_{\delta}\right)\right\|
\end{aligned}
$$

we have

$$
\kappa_{\alpha}\left\|D_{\alpha}^{1 / 2} B_{\alpha}\left(A u_{\alpha}-f_{\delta}\right)\right\| \leq b \delta, \quad \alpha \leq \alpha(\delta) .
$$

Note that for numerical realization of rule R2 additionally iterated approximations $u_{\alpha, m+1}$ and $u_{\alpha, m+2}$ may be replaced by linear combinations of approximations $u_{\alpha, m}$ with different parameters $\alpha$. These ideas for rules MD, ME and $\mathrm{R} 1$ are realized in $[16]$.

\section{Convergence and Order Optimality}

Theorem 1. Let $Q f \in R(A),\left\|f_{\delta}-f\right\| \leq \delta$ and the parameter $\alpha(\delta)$ be chosen by Rule R2 with $b>1$. Then $\left\|u_{\alpha(\delta)}-u_{*}\right\| \rightarrow 0 \quad(\delta \rightarrow 0)$ and in case $u_{0}-u_{*}=$ $|A|^{p} v,\|v\| \leq \varrho$ we have

$$
\left\|u_{\alpha(\delta)}-u_{*}\right\| \leq c \varrho^{1 /(p+1)} \delta^{p /(p+1)}, \quad 0<p \leq 2 m-1 .
$$

To prove this theorem we need the following auxiliary result, which was proved in [15] (Lemma 3). 
Lemma 1. Let $\alpha(\delta)$ be such a parameter that for each $\alpha \leq \alpha(\delta)$ the inequality

$$
\left\|D_{\alpha}^{1 / 2} B_{\alpha}^{2 m+1} A w\right\| \leq c \delta, \quad w \in H, \quad c>0
$$

holds. Then $\alpha(\delta) \leq \alpha_{0}$, where $\alpha_{0}$ is a parameter, for which the function

$$
\varrho(\alpha)=\left\|\bar{B}_{\alpha}^{2 m} w\right\|+2 m c \frac{\delta}{\sqrt{\alpha}}
$$

has a global minimum.

Proof. [Proof of Theorem 1] We introduce notations $\bar{u}:=u_{0}-u_{*}, \bar{f}:=f_{\delta}-f$. From the representation $u_{\alpha}-u_{*}=\bar{B}_{\alpha}^{2 m} \bar{u}+g_{\alpha, m}\left(A^{*} A\right) A^{*} \bar{f}$ and from $(2.2)$ it follows that

$$
\left\|u_{\alpha(\delta)}-u_{*}\right\| \leq\left\|\bar{B}_{\alpha}^{2 m} \bar{u}\right\|+\left\|g_{\alpha(\delta), m}\left(A^{*} A\right) A^{*} \bar{f}\right\| \leq\left\|\bar{B}_{\alpha}^{2 m} \bar{u}\right\|+\gamma_{*} \frac{\delta}{\sqrt{\alpha(\delta)}} .
$$

At first we estimate $\left\|\bar{B}_{\alpha}^{2 m} \bar{u}\right\|$. Since

$$
\begin{aligned}
& D_{\alpha}^{1 / 2} B_{\alpha}\left(A u_{\alpha}-f_{\delta}\right)=D_{\alpha}^{1 / 2} B_{\alpha}^{2 m+1} A \bar{u}-D_{\alpha}^{1 / 2} B_{\alpha}^{2 m+1} \bar{f} \\
& \left\|D_{\alpha}^{1 / 2} B_{\alpha}^{2 m+1} \bar{f}\right\| \leq \delta \sup _{0 \leq \lambda \leq\|A\|^{2}} \frac{\alpha^{(2 m+1) / 2} \lambda^{1 / 2}}{(\alpha+\lambda)^{1 / 2+(2 m+1) / 2}} \leq \widetilde{\gamma}_{1 / 2,2 m+1} \delta,
\end{aligned}
$$

it follows from inequality (2.6) that

$$
\left\|D_{\alpha}^{1 / 2} B_{\alpha}^{2 m+1} A \bar{u}\right\| \leq\left(b \kappa_{\alpha}^{-1}+\widetilde{\gamma}_{1 / 2,2 m+1}\right) \delta \leq\left(b+\widetilde{\gamma}_{1 / 2,2 m+1}\right) \delta, \quad \alpha \leq \alpha(\delta) .
$$

Use of Lemma 1 with $c=b+\widetilde{\gamma}_{1 / 2,2 m+1}, w=\bar{u}$ gives

$$
\left\|\bar{B}_{\alpha(\delta)}^{2 m} \bar{u}\right\| \leq\left\|\bar{B}_{\alpha_{0}}^{2 m} \bar{u}\right\| \leq\left\|\bar{B}_{\alpha_{0}}^{2 m} \bar{u}\right\|+2 m\left(b+\widetilde{\gamma}_{1 / 2,2 m+1}\right) \frac{\delta}{\sqrt{\alpha_{0}}}=\inf _{\alpha \geq 0} \varrho(\alpha) .
$$

Since $\inf _{\alpha \geq 0} \varrho(\alpha) \rightarrow 0$ as $\delta \rightarrow 0$, the convergence $\left\|\bar{B}_{\alpha(\delta)}^{2 m} \bar{u}\right\|$ is proved. If $u_{*}-u_{0}$ has source-like representation, then due to $(2.2),(2.3)$ the estimate

$$
\inf _{\alpha \geq 0} \varrho(\alpha) \leq c_{p} \varrho^{1 /(p+1)} \delta^{p /(p+1)}, \quad 0 \leq p \leq 2 m
$$

is valid. From $(2.5)$ it follows that $\left\|B_{\alpha(\delta)}^{2 m} A \bar{u}\right\| \geq(b-1) \delta$. Analogously to the proof of convergence and order optimality in discrepancy principle (see $[19,20])$ the last inequality gives

$$
\frac{\delta}{\sqrt{\alpha(\delta)}} \rightarrow 0 \quad \text { and } \quad \frac{\delta}{\sqrt{\alpha(\delta)}} \leq d_{p} \varrho^{1 /(p+1)} \delta^{p /(p+1)}, \quad 0 \leq p \leq 2 m-1 .
$$

We note that numerical examples and results of Section 4 show that probably the estimate $(3.1)$ holds with $0<p \leq 2 m$. 


\section{On the Quasioptimality of Rule R2}

In this section we show that the choice of $\alpha$ by rule $\mathrm{R} 2$ in methods $(1.2),(1.3)$ is under certain conditions weakly quasioptimal (see [15]) in sense that the error estimate (3.2) also holds, if the right hand side there is replaced by its infimum over $\alpha \geq 0$, multiplied by a constant.

We denote

$$
t_{c}(\alpha):=\left\|\bar{B}_{\alpha}^{2 m} \bar{u}\right\|^{2}+c\left\|g_{\alpha, m}\left(A^{*} A\right) A^{*} \bar{f}\right\|^{2} .
$$

Let $\left\{F_{\lambda}\right\}$ denote the spectral family of the operator $A A^{*}$. From (2.1) follows that

$$
\begin{aligned}
& \frac{d}{d \alpha}\left\|\bar{B}_{\alpha}^{2 m} \bar{u}\right\|^{2}=\frac{d}{d \alpha}\left(\left(\alpha^{-1} A^{*} A+I\right)^{-2 m} \bar{u}, \bar{u}\right)=\frac{d}{d \alpha} \int_{0}^{\left\|A^{*} A\right\|}\left(\alpha^{-1} \lambda+1\right)^{-2 m} d\left\|F_{\lambda} \bar{u}\right\|^{2} \\
& =\frac{2 m}{\alpha^{2}} \int_{0}^{\left\|A^{*} A\right\|}\left(\alpha^{-1} \lambda+1\right)^{-2 m-1} d\left\|F_{\lambda} \bar{u}\right\|^{2}=\frac{2 m}{\alpha^{2}}\left(A^{*} A\left(\alpha^{-1} A^{*} A+I\right)^{-2 m-1} \bar{u}, \bar{u}\right) \\
& =\frac{2 m}{\alpha^{2}}\left\|B_{\alpha}^{2 m+1} A \bar{u}\right\|^{2} .
\end{aligned}
$$

\section{Lemma 2. If}

$$
\kappa_{\alpha}^{3 / 2}\left\|D_{\alpha}^{1 / 2} B_{\alpha}^{2 m+1} A \bar{u}\right\| \geq \sqrt{c m}\left\|D_{\alpha}^{1 / 4} B_{\alpha}^{1+m} \bar{f}\right\|, \quad \alpha \in\left[\alpha_{0}, \alpha_{1}\right]
$$

then the function $t_{c}(\alpha)$ is monotonically increasing in the interval $\left[\alpha_{0}, \alpha_{1}\right]$.

Proof. From (2.1) follows that $\frac{d}{d \alpha} g_{\alpha, m}(\lambda)=-\frac{m}{\alpha^{2}}\left(\frac{\alpha}{\alpha+\lambda}\right)^{m+1}$. Using this equality and the equality $g_{\alpha, m}\left(A^{*} A\right) A^{*}=A^{*} g_{\alpha, m}\left(A A^{*}\right)($ see $[19,20])$ we have

$$
\frac{d g_{\alpha, m}\left(A^{*} A\right) A^{*} \bar{f}}{d \alpha}=\frac{A^{*} d g_{\alpha, m}\left(A A^{*}\right) \bar{f}}{d \alpha}=-\frac{m}{\alpha^{2}} A^{*} B_{\alpha}^{2 m+2} \bar{f} .
$$

This equality and the inequality $g_{\alpha, m}(\lambda) \leq \frac{m}{\alpha}\left(\frac{\alpha}{\alpha+\lambda}\right)$ (see $\left.(2.1)\right)$ give

$$
\begin{aligned}
\frac{1}{2} \frac{d}{d \alpha} & \left\|g_{\alpha, m}\left(A^{*} A\right) A^{*} \bar{f}\right\|^{2}=\left(g_{\alpha, m}\left(A^{*} A\right) A^{*} \bar{f}, \frac{d g_{\alpha, m}\left(A^{*} A\right) A^{*} \bar{f}}{d \alpha}\right) \\
& =-\frac{m}{\alpha^{2}}\left(A^{*} g_{\alpha, m}\left(A A^{*}\right) \bar{f}, A^{*} B_{\alpha}^{2 m+2} \bar{f}\right)=-\frac{m}{\alpha^{2}}\left\|A^{*} g_{\alpha, m}^{1 / 2}\left(A A^{*}\right) B_{\alpha}^{m+1} \bar{f}\right\|^{2} \\
& \geq-\frac{m^{2}}{\alpha^{3}}\left\|A^{*} B_{\alpha}^{2+m} \bar{f}\right\|^{2} .
\end{aligned}
$$

This inequality and equality (4.1) give

$$
\frac{1}{2} t_{c}^{\prime}(\alpha) \geq \frac{m}{\alpha^{2}}\left[\left\|B_{\alpha}^{2 m+1} A \bar{u}\right\|^{2}-\frac{c m}{\alpha}\left\|A^{*} B_{\alpha}^{2+m} \bar{f}\right\|^{2}\right] .
$$


Since $\left\|\alpha^{-1}\left|A^{*}\right|^{2} B_{\alpha}^{2}\right\| \leq \kappa_{\alpha}^{-2}$ we have

$$
\begin{aligned}
t_{c}^{\prime}(\alpha) & \geq \frac{2 m}{\alpha^{2}}\left[\kappa_{\alpha}^{2}\left\|\left(\frac{1}{\alpha}\left|A^{*}\right|^{2} B_{\alpha}^{2}\right)^{1 / 2} B_{\alpha}^{2 m+1} A \bar{u}\right\|^{2}-\frac{c m}{\alpha}\left\|\left|A^{*}\right| B_{\alpha}^{2+m} \bar{f}\right\|^{2}\right] \\
& \geq \frac{2 m}{\alpha^{2}}\left[\kappa_{\alpha}^{2}\left\|D_{\alpha}^{1 / 2} B_{\alpha}^{2 m+1} A \bar{u}\right\|^{2}-\kappa_{\alpha}^{-1} c m\left\|D_{\alpha}^{1 / 4} B_{\alpha}^{1+m} \bar{f}\right\|^{2}\right] .
\end{aligned}
$$

Therefore in case (4.2) the function $t_{c}(\alpha)$ monotonically increases in the interval $\left[\alpha_{0}, \alpha_{1}\right]$.

In the following, we need the condition

$$
\begin{aligned}
\left\|D_{\alpha}^{1 / 2} B_{\alpha}^{2}\left(A u_{\alpha}-f_{\delta}\right)\right\| \equiv\left\|D_{\alpha}^{1 / 2} B_{\alpha}^{2+2 m} A \bar{u}-D_{\alpha}^{1 / 2} B_{\alpha}^{2+2 m} \bar{f}\right\| & \geq\left\|D_{\alpha}^{1 / 2} B_{\alpha}^{2+2 m} \bar{f}\right\| \\
& \forall \alpha \geq \alpha(\delta) .
\end{aligned}
$$

This condition is satisfied in special cases, if the error of the right hand side $\bar{f}=f_{\delta}-f$ is such that $\left(F_{\lambda} \bar{u}, \bar{f}\right) \leq 0, \forall \lambda \geq 0$. Numerical experiments show that for most severely ill-posed problems in case of uniform distribution of error $\bar{f}$ the condition $\left\|D_{\alpha}^{1 / 2} B_{\alpha}^{2}\left(A u_{\alpha}-f_{\delta}\right)\right\| \geq c_{*}\left\|D_{\alpha}^{1 / 2} B_{\alpha}^{2 m+2} \bar{f}\right\|$ is fulfilled with constant $c_{*} \approx 1$.

Lemma 3. If condition (4.3) holds and for $\alpha \geq \alpha(\delta)$ the equation (2.4) has the unique solution with $b>2$ then the function $t_{c}(\alpha)$ with $c=\frac{1}{m}\left(\frac{b}{2}-1\right)$ is monotonically increasing for $\alpha \geq \alpha(\delta)$.

Proof. From the equality

$$
D_{\alpha}^{1 / 2} B_{\alpha}\left(A u_{\alpha}-f_{\delta}\right)=D_{\alpha}^{1 / 2} B_{\alpha}^{2 m+1} A \bar{u}-D_{\alpha}^{1 / 2} B_{\alpha}^{2 m+1} \bar{f}
$$

it follows that

$$
\left\|D_{\alpha}^{1 / 2} B_{\alpha}\left(A u_{\alpha}-f_{\delta}\right)\right\|^{2} \leq 2\left(\left\|D_{\alpha}^{1 / 2} B_{\alpha}^{2 m+1} A \bar{u}\right\|^{2}+\left\|D_{\alpha}^{1 / 2} B_{\alpha}^{2 m+1} \bar{f}\right\|^{2}\right) .
$$

This inequality together with condition (4.3) gives

$d(\alpha)=\frac{\kappa_{\alpha}\left\|D_{\alpha}^{1 / 2} B_{\alpha}\left(A u_{\alpha}-f_{\delta}\right)\right\|^{2}}{\left\|D_{\alpha}^{1 / 2} B_{\alpha}^{2}\left(A u_{\alpha}-f_{\delta}\right)\right\|} \leq \frac{2 \kappa_{\alpha}\left(\left\|D_{\alpha}^{1 / 2} B_{\alpha}^{2 m+1} A \bar{u}\right\|^{2}+\left\|D_{\alpha}^{1 / 2} B_{\alpha}^{2 m+1} \bar{f}\right\|^{2}\right)}{\left\|D_{\alpha}^{1 / 2} B_{\alpha}^{2 m+2} \bar{f}\right\|}$.

Assume now that $\alpha \geq \alpha(\delta)$. Then from (2.4), (4.4) we conclude that

$$
\kappa_{\alpha}\left\|D_{\alpha}^{1 / 2} B_{\alpha}^{2 m+1} A \bar{u}\right\|^{2} \geq \frac{b}{2} \delta\left\|D_{\alpha}^{1 / 2} B_{\alpha}^{2 m+2} \bar{f}\right\|-\kappa_{\alpha}\left\|D_{\alpha}^{1 / 2} B_{\alpha}^{2 m+1} \bar{f}\right\|^{2} .
$$

To continue this estimation, we use the inequalities $\left\|B_{\alpha}\right\| \leq 1,\left\|D_{\alpha}\right\| \leq 1$,

$$
\begin{aligned}
& \kappa_{\alpha}^{1 / 2}\left\|D_{\alpha}^{1 / 2} B_{\alpha}^{2 m+1} \bar{f}\right\| \leq \kappa_{\alpha}^{1 / 2}\left\|D_{\alpha}^{1 / 4} B_{\alpha}^{m}\right\|\left\|D_{\alpha}^{1 / 4} B_{\alpha}^{m+1} \bar{f}\right\| \leq\left\|D_{\alpha}^{1 / 4} B_{\alpha}^{m+1} \bar{f}\right\|, \\
& \left\|D_{\alpha}^{1 / 4} B_{\alpha}^{m+1} \bar{f}\right\|^{2}=\left(D_{\alpha}^{1 / 2} B_{\alpha}^{2 m+2} \bar{f}, \bar{f}\right) \leq\left\|D_{\alpha}^{1 / 2} B_{\alpha}^{2 m+2} \bar{f}\right\|\|\bar{f}\| \leq\left\|D_{\alpha}^{1 / 2} B_{\alpha}^{2 m+2} \bar{f}\right\| \delta
\end{aligned}
$$


and get

$$
\kappa_{\alpha}^{1 / 2}\left\|D_{\alpha}^{1 / 2} B_{\alpha}^{2 m+1} A \bar{u}\right\| \geq \sqrt{b / 2-1}\left\|D_{\alpha}^{1 / 4} B_{\alpha}^{1+m} \bar{f}\right\| .
$$

The monotonical increase of the function $t_{c}(\alpha)$ with $c=\frac{1}{m}\left(\frac{b}{2}-1\right)$ follows now from Lemma 2.

Theorem 2. Let $Q f \in R(A),\left\|f_{\delta}-f\right\| \leq \delta$. Let the condition (4.3) hold and let the parameter $\alpha \geq \alpha(\delta)$ be the unique solution of the equation (2.4) with $b>2$. Then the quasioptimal error estimate

$$
\left\|u_{\alpha(\delta)}-u_{*}\right\| \leq C(b, m) \inf _{\alpha \geq 0} \psi(\alpha)
$$

holds, where $\psi(\alpha)=\left\|\bar{B}_{\alpha}^{2 m} \bar{u}\right\|+\gamma_{*} \frac{\delta}{\sqrt{\alpha}}$,

$$
C(b, m)=2 \sqrt{m}\left(b+\widetilde{\gamma}_{1 / 2,2 m+1}\right)+\sqrt{\max \left(\frac{m}{b / 2-1}, \frac{b / 2-1}{m}\right)} .
$$

Proof. We denote by $\alpha_{*}$ the global minimum point of the function $t_{c}(\alpha)$ with $c=\frac{1}{m}\left(\frac{b}{2}-1\right)$. If $\alpha(\delta)$ is the unique solution of the equation $(2.4)$ then according to Lemma $3 \alpha(\delta) \geq \alpha_{*}$. From the representation

$$
u_{\alpha}-u_{*}=\bar{B}_{\alpha}^{2 m} \bar{u}+g_{\alpha, m}\left(A^{*} A\right) A^{*} \bar{f}
$$

it follows that

$$
\left\|u_{\alpha(\delta)}-u_{*}\right\| \leq\left\|\bar{B}_{\alpha(\delta)}^{2 m} \bar{u}\right\|+\left\|g_{\alpha(\delta), m}\left(A^{*} A\right) A^{*} \bar{f}\right\| .
$$

Denoting $\underline{c}=\min (c, 1)$ and $\bar{c}=\max (c, 1)$ we have

$$
\bar{c}^{-1} t_{c}(\alpha) \leq\left\|\bar{B}_{\alpha}^{2 m} \bar{u}\right\|^{2}+\left\|g_{\alpha, m}\left(A^{*} A\right) A^{*} \bar{f}\right\|^{2} \leq \psi^{2}((\alpha),
$$

therefore

$$
\left\|g_{\alpha(\delta), m}\left(A^{*} A\right) A^{*} \bar{f}\right\| \leq\left\|g_{\alpha_{*}, m}\left(A^{*} A\right) A^{*} \bar{f}\right\| \leq \sqrt{\frac{t_{c}\left(\alpha_{*}\right)}{\underline{c}}} \leq \sqrt{\frac{\bar{c}}{\underline{c}} \inf _{\alpha \geq 0} \psi(\alpha) .}
$$

To estimate the first summand in (4.6), we use the inequality (2.6) which allows us to use Lemma 1 with $c=b \kappa_{\alpha}^{-1}+\widetilde{\gamma}_{1 / 2,2 m+1}, w=\bar{u}$ and we get

$$
\begin{aligned}
\left\|\bar{B}_{\alpha(\delta)}^{2 m} \bar{u}\right\| & \leq\left\|\bar{B}_{\alpha_{0}}^{2 m} \bar{u}\right\| \leq\left\|\bar{B}_{\alpha_{0}}^{2 m} \bar{u}\right\|+2 m\left(b+\widetilde{\gamma}_{1 / 2,2 m+1}\right) \frac{\delta}{\sqrt{\alpha_{0}}} \\
& =\inf _{\alpha \geq 0}\left[\left\|\bar{B}_{\alpha}^{2 m} \bar{u}\right\|+2 m\left(b+\widetilde{\gamma}_{1 / 2,2 m+1}\right) \frac{\delta}{\sqrt{\alpha}}\right] \\
& \leq 2 \sqrt{m}\left(b+\widetilde{\gamma}_{1 / 2,2 m+1}\right) \inf _{\alpha \geq 0} \psi(\alpha) .
\end{aligned}
$$


Summation of the last inequality and (4.7) gives asserted estimate (4.5).

The quasioptimality of the rule (2.4) is proved under the special condition (4.3) for $\bar{f}$, but remains an open problem in the general case. Note, that quasioptimality guarantees best convergence rates on all solution sets, including sets from $[5,6,7,9,10,17]$.

The results of Theorems 1, 2 were proved for constant $b>2$. The following Theorem 3 shows, that under certain additional conditions the quasioptimality of Rule R2 holds also for smaller $b$. Numerical experiments show that for constant $b$ smaller values than indicated in Rule R2 may be used.

Theorem 3. Let $Q f \in R(A),\left\|f_{\delta}-f\right\| \leq \delta$. Let $\alpha=\alpha(\delta)$ be chosen by Rule R2. Let the inequality $d(\alpha) \geq b \delta, b \geq 2 \kappa_{\alpha_{1}} \widetilde{\gamma}_{1 / 4, m+1}^{2}, \alpha \in\left[\alpha(\delta), \alpha_{1}\right]$ hold, where $\alpha_{1}$ is the parameter, for which $\left\|B_{\alpha}\left(A u_{\alpha}-f_{\delta}\right)\right\|=\left(1+\sqrt{\gamma_{*} \widetilde{\gamma}_{1 / 2,2 m+1}}\right) \delta$. Then

$$
\left\|u_{\alpha(\delta)}-u_{*}\right\| \leq C(b, m) \inf _{\alpha \geq 0} \psi(\alpha) .
$$

\section{$5 \quad$ Numerical Experiments}

We solved test problems from [4] with discretization parameter 100, the other parameters had standard values. Instead of exact data $f$, a randomly perturbed data $f_{\delta}$ was used with error $\left\|f-f_{\delta}\right\|=d \delta$, where the values of $\delta$ were $10^{-(i+1) / 2}\|f\|, i=1, \ldots, 11$ and the values of $d$ were $1,0.7$ and 0.3 . In case $d<1$ the noise was overestimated: actual noise $\left\|f-f_{\delta}\right\|$ was smaller than its estimate $\delta$. The problems were regularized by the Tikhonov method using $\alpha$ values $\alpha_{i}=q^{i}, i=0,1, \ldots, q=0.9$. In the model equations the exact solutions are known. We found $\alpha_{\text {opt }}$ as $\alpha$ with the smallest error:

$$
\left\|u_{\alpha_{o p t}}-u_{*}\right\|=\min _{i}\left\{\left\|u_{\alpha_{i}}-u_{*}\right\|\right\} .
$$

We solved these problems 10 times using ME rule with constant $b=1.01$, the MEE rule and rule R2 with constants $b=b 1:=1.01 \widetilde{\gamma}_{1 / 4,1}^{2}=1.01 \frac{2}{3 \sqrt{3}}$ and $b=b 2:=0.7 \widetilde{\gamma}_{1 / 4,1}^{2}$. We also used the combination of the ME and R2 rules with $b=b 2$ taking the minimal value from these parameters: $\alpha_{M E R 2}(\delta)=$ $\min \left\{\alpha_{M E}(\delta), \alpha_{R 2}(\delta)\right\}$. In Tables 1-3 the averages and maxima (over all $\delta$ and 10 runs) of the error ratios $r=\left\|u_{\alpha(\delta)}-u_{*}\right\| /\left\|u_{\alpha_{o p t}}-u_{*}\right\|$ are given with parameter in numerator from the corresponding rule.

In case of exactly given noise level $\delta$ (see Table 1 ) the MEE rule gave the best results, the rule R2 with $b=b 1$ gave worse results than the ME rule and the rule R2 with $b=b 2$ gave in half of our problems better results than the $\mathrm{ME}$ rule. The combination of the $\mathrm{ME}$ and $\mathrm{R} 2$ rules gave better results than $\mathrm{ME}$ and $\mathrm{R} 2$ rules. In case of overestimated $\delta$ (see Tables 2,3 ) the rule R2 with both constants $b$ is better than the ME rule and MEE rule (if $d>1$, parameter $\alpha_{M E}^{c}$ with $c>1.1$ would be better than $\left.\alpha_{M E}^{1.1}\right)$ and gave in most cases a smaller parameter than the ME rule.

We recommend the new rule (or its combination with ME rule or with MEE rule) especially in case of roughly estimated noise level $\delta$. 
Table 1. Error ratios in case of $\left\|f-f_{\delta}\right\|=\delta$.

\begin{tabular}{lrrrrrrrrrr}
\hline & \multicolumn{2}{c}{ ME } & \multicolumn{2}{c}{ MEE } & \multicolumn{2}{c}{$\mathrm{R} 2$} & $\mathrm{~b}=\mathrm{b} 1$ & $\mathrm{R} 2$ & $\mathrm{~b}=\mathrm{b} 2$ & \multicolumn{2}{c}{$\mathrm{R} 2 \& \mathrm{ME}$} \\
\hline Problem & Avg & Max & Avg & Max & Avg & Max & Avg & Max & Avg & Max \\
\hline baart & 1,54 & 2,49 & 1,42 & 2,32 & 1,83 & 3,00 & 1,66 & 2,93 & 1,53 & 2,49 \\
baart2 & 3,02 & 9,43 & 1,99 & 6,73 & 3,14 & 12,81 & 2,51 & 9,32 & 2,43 & 9,32 \\
deriv2 & 1,13 & 1,49 & 1,03 & 1,25 & 1,11 & 1,33 & 1,05 & 1,20 & 1,05 & 1,20 \\
foxgood & 2,40 & 11,34 & 1,68 & 9,23 & 3,40 & 20,41 & 2,85 & 19,08 & 2,32 & 11,34 \\
gravity & 1,44 & 2,07 & 1,26 & 1,88 & 1,56 & 2,49 & 1,37 & 2,07 & 1,35 & 1,99 \\
heat & 1,21 & 1,40 & 1,02 & 1,14 & 1,13 & 1,56 & 1,04 & 1,22 & 1,04 & 1,22 \\
heat2 & 1,22 & 1,55 & 1,12 & 1,33 & 1,05 & 1,55 & 1,03 & 1,20 & 1,03 & 1,20 \\
ilaplace & 1,26 & 2,06 & 1,16 & 1,67 & 1,34 & 2,30 & 1,27 & 2,14 & 1,25 & 2,05 \\
phillips & 1,31 & 2,32 & 1,20 & 2,11 & 1,25 & 1,96 & 1,14 & 1,76 & 1,14 & 1,76 \\
shaw & 1,56 & 4,56 & 1,41 & 4,19 & 1,73 & 4,56 & 1,61 & 4,54 & 1,52 & 4,54 \\
spikes & 1,01 & 1,02 & 1,01 & 1,02 & 1,01 & 1,05 & 1,01 & 1,04 & 1,01 & 1,02 \\
wing & 1,21 & 1,66 & 1,17 & 1,66 & 1,27 & 1,77 & 1,24 & 1,77 & 1,20 & 1,66 \\
\hline average & 1,52 & 3,45 & 1,29 & 2,88 & 1,65 & 4,57 & 1,48 & 4,02 & 1,41 & 3,32 \\
\hline
\end{tabular}

Table 2. Error ratios in case of $\left\|f-f_{\delta}\right\|=0.7 \delta$.

\begin{tabular}{|c|c|c|c|c|c|c|c|c|c|c|}
\hline \multirow[b]{2}{*}{ Problem } & \multicolumn{2}{|c|}{$\mathrm{ME}$} & \multicolumn{2}{|c|}{$\mathrm{MEE}$} & \multirow{2}{*}{$\frac{\mathrm{R} 2}{\mathrm{Avg}}$} & \multirow{2}{*}{$\frac{\mathrm{b}=\mathrm{b} 1}{\operatorname{Max}}$} & \multirow{2}{*}{$\frac{\mathrm{R} 2}{\mathrm{Avg}}$} & \multirow{2}{*}{$\frac{\mathrm{b}=\mathrm{b} 2}{\operatorname{Max}}$} & \multicolumn{2}{|c|}{$\mathrm{R} 2 \& \mathrm{ME}$} \\
\hline & Avg & Max & Avg & Max & & & & & Avg & $\operatorname{Max}$ \\
\hline baart & 2,16 & 3,22 & 2,00 & 3,11 & 2,01 & 3,10 & 1,84 & 3,07 & 1,84 & 3,07 \\
\hline baart2 & 8,32 & 28,26 & 5,76 & 23,13 & 4,08 & 18,90 & 3,25 & 17,13 & 3,25 & 17,1 \\
\hline Deriv2 & 1,75 & 4,07 & 1,19 & 2,28 & 1,16 & 1,61 & 1,08 & 1,37 & 1,08 & 1,37 \\
\hline foxgood & 5,12 & 19,74 & 3,83 & 18,73 & 3,80 & 17,06 & 3,20 & 15,88 & 3,20 & 15,9 \\
\hline gravity & 2,57 & 5,17 & 2,31 & 4,49 & 1,96 & 4,07 & 1,70 & 3,46 & 1,70 & 3,46 \\
\hline Heat & 1,58 & 2,17 & 1,18 & 1,85 & 1,25 & 1,82 & 1,11 & 1,36 & 1,11 & 1,36 \\
\hline Heat2 & 1,80 & 2,71 & 1,12 & 1,91 & 1,12 & 1,78 & 1,04 & 1,32 & 1,04 & 1,32 \\
\hline ilaplace & 1,68 & 3,92 & 1,54 & 3,24 & 1,56 & 3,65 & 1,48 & 3,42 & 1,48 & 3,42 \\
\hline phillips & 2,02 & 4,33 & 1,83 & 4,67 & 1,41 & 2,43 & 1,25 & 1,97 & 1,25 & 1,97 \\
\hline shaw & 1,95 & 3,50 & 1,81 & 3,36 & 1,76 & 2,91 & 1,64 & 2,79 & 1,64 & 2,79 \\
\hline spikes & 1,01 & 1,06 & 1,01 & 1,06 & 1,01 & 1,06 & 1,01 & 1,03 & 1,01 & 1,03 \\
\hline wing & 1,27 & 1,78 & 1,24 & 1,78 & 1,25 & 1,78 & 1,24 & 1,78 & 1,24 & 1,78 \\
\hline average & 2,60 & 6,66 & 2,07 & 5,80 & 1,86 & 5,01 & 1,65 & 4,55 & 1,65 & 4,55 \\
\hline & \multicolumn{2}{|c|}{$\mathrm{ME}$} & \multicolumn{2}{|c|}{ MEE } & \multicolumn{2}{|c|}{$\mathrm{R} 2 \mathrm{~b}$} & \multicolumn{2}{|c|}{$\mathrm{R} 2 \quad \mathrm{~b}=\mathrm{b} 2$} & \multicolumn{2}{|c|}{$\mathrm{R} 2 \& \mathrm{ME}$} \\
\hline Problem & Avg & Max & Avg & $\operatorname{Max}$ & Avg & Max & Avg & Max & Avg & Max \\
\hline baart & 2,32 & 4,40 & 2,18 & 4,34 & 2,07 & 3,38 & 1,87 & 3,31 & 1,87 & 3,31 \\
\hline baart2 & 13,52 & 62,25 & 9,22 & 28,88 & 6,17 & 22,10 & 5,03 & 20,66 & 5,03 & 20,7 \\
\hline Deriv2 & 2,45 & 8,17 & 1,58 & 4,50 & 1,39 & 2,29 & 1,28 & 1,74 & 1,28 & 1,74 \\
\hline foxgood & 8,69 & 35,08 & 6,60 & 26,07 & 5,71 & 22,61 & 4,71 & 21,59 & 4,71 & 21,6 \\
\hline gravity & 3,59 & 7,41 & 3,28 & 8,09 & 2,58 & 4,55 & 2,26 & 3,91 & 2,26 & 3,91 \\
\hline Heat & 2,19 & 3,35 & 1,58 & 2,81 & 1,62 & 2,35 & 1,39 & 1,80 & 1,39 & 1,80 \\
\hline Heat2 & 2,92 & 5,04 & 1,49 & 3,92 & 1,50 & 3,18 & 1,28 & 2,48 & 1,28 & 2,48 \\
\hline ilaplace & 1,89 & 3,52 & 1,74 & 3,06 & 1,69 & 3,18 & 1,61 & 3,01 & 1,61 & 3,01 \\
\hline phillips & 2,83 & 5,38 & 2,55 & 5,80 & 1,84 & 3,07 & 1,60 & 2,55 & 1,60 & 2,55 \\
\hline shaw & 2,30 & 4,64 & 2,13 & 4,17 & 1,99 & 4,19 & 1,85 & 4,11 & 1,85 & 4,11 \\
\hline spikes & 1,02 & 1,05 & 1,02 & 1,05 & 1,01 & 1,05 & 1,01 & 1,03 & 1,01 & 1,03 \\
\hline wing & 1,29 & 1,82 & 1,25 & 1,82 & 1,25 & 1,82 & 1,24 & 1,82 & 1,25 & 1,82 \\
\hline average & 3,75 & 11,84 & 2,89 & 7,88 & 2,40 & 6,15 & 2,10 & 5,67 & 2,10 & 5,67 \\
\hline
\end{tabular}




\section{References}

[1] H. Gfrerer. An a posteriori parameter choice for ordinary and iterated Tikhonov regularization of ill-posed problems leading to optimal convergence rates. Math. Comp., 49(180):507-522, 1987.

[2] U. Hämarik, R. Palm and T. Raus. Extrapolation of Tikhonov and Lavrentiev regularization methods. Journal of Physics: Conference Series, 135:012087, 2008.

[3] U. Hämarik and T. Raus. On the choice of the regularization parameter in illposed problems with approximately given noise level of data. Journal of Inverse and Ill-Posed Problems, 14(3):251-266, 2006.

[4] P. C. Hansen. Regularization tools: A matlab package for analysis and solution of discrete ill-posed problems. Numerical Algorithms, 6(1):1-35, 1994.

[5] B. Hofmann, D. Düvelmeyer and K. Krumbiegel. Approximate source conditions in Tikhonov regularization - new analytical results and some numerical studies. Math. Model. Anal., 11(1):41-56, 2006.

[6] T. Hohage. Regularization of exponentially ill-posed problems. Numerical Functional Analysis and Optimization, 21(3):439-464, 2000.

[7] P. Mathé and S. V. Pereverzev. Geometry of linear ill-posed problems in variable Hilbert scales. Inverse Problems, 19(3):789-803, 2003.

[8] V. A. Morozov. On the solution of functional equations by the method of regularization. Soviet Math. Dokl., 7:414-417, 1966.

[9] M. T. Nair, S. V. Pereverzev and U. Tautenhahn. Regularization in Hilbert scales under general smoothing conditions. Inverse Problems, 21(6):1851-1869, 2005.

[10] S. V. Pereverzev and E. Schock. On the adaptive selection of the parameter in the regularization of ill-posed problems. SIAM J. Numerical Analysis, 43(5):20602076, 2005.

[11] T. Raus. Residue principle for ill-posed problems. Acta et Comment. Univ. Tartuensis, 672:16-26, 1984. (in Russian)

[12] T. Raus. On the discrepancy principle for solution of ill-posed problems with nonselfadjoint operators. Acta et Comment. Univ. Tartuensis, 715:12-20, 1985. (in Russian)

[13] T. Raus. An a posteriori choice of the regularization parameter in case of approximately given error bound of data. Acta et Comment. Univ. Tartuensis, 913:73-87, 1990.

[14] T. Raus. About regularization parameter choice in case of approximately given error bounds of data. Acta et Comment. Univ. Tartuensis, 937:77-89, 1992.

[15] T. Raus and U. Hämarik. On the quasioptimal regularization parameter choices for solving ill-posed problems. Journal of Inverse and Ill-Posed Problems, 15(4):419-439, 2007.

[16] T. Raus and U. Hämarik. On numerical realization of quasioptimal parameter choices in (iterated) Tikhonov and Lavrentiev regularization. Math. Model. Anal., 14(1):99-108, 2009. (doi:10.3846/1392-6292.2009.14.99-108)

[17] U. Tautenhahn. Optimality for ill-posed problems under general source conditions. Numer. Funct. Anal. Optim., 19(3-4):377-398, 1998. 
[18] U. Tautenhahn and U. Hämarik. The use of monotonicity for choosing the regularization parameter in ill-posed problems. Inverse Problems, 15(6):1487 U$1505,1999$.

[19] G. Vainikko and A. Veretennikov. Iteration Procedures in Ill-Posed Problems. Nauka, Moscow, 1986. (in Russian)

[20] G. M. Vainikko. The principle of the residual for a class of regularization methods. USSR Comput. Math. Math. Phys., 22(3):1-19, 1982. 